\title{
Action potentials contribute to epileptic high-frequency oscillations recorded with electrodes remote from neurons
}

\author{
Katsuhiro Kobayashi, ${ }^{1}$ Tomoyuki Akiyama, ${ }^{1}$ Iori Ohmori, ${ }^{2}$ Harumi Yoshinaga, ${ }^{1}$ \\ Jean Gotman ${ }^{3}$
}

\footnotetext{
${ }^{1}$ Department of Child Neurology, and ${ }^{2}$ Department of Physiology, Okayama University Graduate School of Medicine, Dentistry and Pharmaceutical Sciences and Okayama University Hospital, Shikatacho 2-chome 5-1, Kita-ku, Okayama 700-8558, Japan

${ }^{3}$ Montreal Neurological Institute, McGill University, 3801 University Street, Montreal, Que. H3A 2B4, Canada
}

\section{Correspondence:}

Katsuhiro Kobayashi

Department of Child Neurology, Okayama University Graduate School of Medicine, Dentistry and Pharmaceutical Sciences, and Okayama University Hospital, Shikatacho 2-chome 5-1, Kita-ku, Okayama 700-8558, Japan

Tel: +81-86-235-7372

Fax: +81-86-235-7377

E-mail: $\underline{\text { _knoba@md.okayama-u.ac.jp }}$ 


\section{Acknowledgements}

We thank Drs. Massimo Avoli and George Kostopoulos of McGill University, Montreal Neurological Institute for important suggestions.

K. Kobayashi was supported by the Research Grant (24-7) for Nervous and Mental Disorders from the Ministry of Health and Welfare, by the Health and Labour Sciences Research Grant: Research on catastrophic epilepsy in infancy and early childhood-epidemiology, diagnosis and treatment guide, and by a Grant-in-Aid from the Ministry of Education, Culture, Sports, Science, and Technology, Japan (No. 24591513).

I. Ohmori was supported by grants from Japan Science and Technology Agency, and grants from the Japanese Ministry of Education, Culture, Sports, Science and Technology (No. 23659522). H. Yoshinaga was supported in part by the Japan Epilepsy Research Foundation. 
Kobayashi et al.: CLINPH-D-14-7633R1 3 


\begin{abstract}
Objective: The importance of epileptic high-frequency oscillations (HFOs) in electroencephalogram (EEG) is growing. Action potentials generating some HFOs are observed in the vicinity of neurons in experimental animals. However electrodes that are remote from neurons, as in case of clinical situations, should not record action potentials. We propose to resolve this question by a realistic simulation of epileptic neuronal network.
\end{abstract}

Methods: The rat dentate gyrus with sclerosis was simulated in silico. We computed the current dipole moment generated by each granule cell and the field potentials in a measurement area far from neurons.

Results: The dentate gyrus was stimulated through synaptic input to evoke discharges resembling interictal epileptiform discharges, which had superimposed HFOs $\leq 295 \mathrm{~Hz}$ that were recordable with remote electrodes and represented bursts of action potentials of granule cells. The increase in power of HFOs was associated with the progression of sclerosis, the reduction of GABAergic inhibition, and the increase in cell connectivity. Spectral frequency of HFOs had similar tendencies.

Conclusions: HFOs recorded with electrodes remote from neurons could actually be generated by clusters of action potentials.

Significance: The phenomenon of action potentials recorded with remote electrodes can possibly extend the clinical meaning of EEG. 
Keywords: High-frequency oscillations; electrode; epilepsy; simulation; dentate gyrus. 


\section{INTRODUCTION}

The importance of high-frequency oscillations (HFOs), particularly fast ripples $>200$ $\mathrm{Hz}$, in the depth electroencephalogram (EEG) is growing ever since the discovery of HFOs recorded with depth electrodes (Jirsch et al. 2006). Evidence is now accumulating to indicate that pathologic HFOs in EEG have a closer relationship with epileptogenicity and/or ictogenicity than interictal epileptiform discharges (IEDs) or “epileptic spikes" have (Engel et al. 2009; Jacobs et al. 2010). However we still do not know the generative mechanisms of HFOs and the exact meaning of HFOs.

Experimental pathologic HFOs recorded with microelectrodes are thought to be generated by action potentials of synchronously bursting principal cells and therefore represent recurrent "population spikes" (Bragin et al. 1999, 2011; Dzhala and Staley 2004; Ibarz et al. 2010). Depth electrodes, however, are generally remote from neurons. This suggestion raises the question of whether action potentials can be really recorded with electrodes far from neurons, and this is even more questionable with reports that fast ripples are generated through the loss of correlation of cell activity (Foffani et al. 2007; Demont-Guignard et al. 2012), which indicates that amplitude of summated activity should be very low. Depth EEG is believed to represent the field potentials generated from neuronal membrane potentials, particularly post-synaptic potentials (PSPs) at dendrites, and not action potentials (Menendez de la Prida et al. 2011). It is believed that fields generated by action potentials are visible only at approximately 10 $\mu \mathrm{m}$ from the generator, a distance much smaller than that between a depth electrode and any significant neuronal assembly (Bédard et al. 2006). The correspondence between action potentials and HFOs in local field potentials (LFP) has been studied only with 
microelectrodes (Bragin et al. 1999, 2011; Dzhala and Staley 2004; Foffani et al. 2007; Ibarz et al. 2010). The reports of lack of relationship between electrode-size and HFO detection were regarding depth electrodes (Châtillon et al. 2011, 2013), and such relationship is still open with respect to microelectrodes. We hypothesize that at least part of the action potentials related to the generation of HFOs may contribute to remote potentials, which is so far unproved to our knowledge.

The behavior of individual neurons has been investigated by intracellular and extracellular recording. However, the relationship between neuronal membrane potentials and depth EEG findings is not easy to comprehend, as EEG recorded with electrodes remote from neurons represents summated field potentials with a considerably large spatial extent including innumerable neurons. Even when some part of an electrode is close to cells, the larger part of electrode is far away from those cells due to the size of the electrode. As computer simulation of neuronal network can be useful and possibly the only way to understand the correspondence between depth EEG discharges and behaviors of the whole neuronal network, we tried to address the above questions, particularly what clinical HFOs represent, through a realistic simulation of neuronal network. Although there are several simulation studies on LFP recorded with microelectrodes placed in the vicinity of neurons (Gold et al. 2006; Ibarz et al. 2010; Schomburg et al. 2012; Wendling et al. 2012; Buzsáki et al. 2012; Reimann et al. 2013), the simulation of fields seen through electrodes far from neurons has not yet been attempted.

The rat dentate gyrus is appropriate for this purpose. Pathological HFOs were observed with microelectrodes in association with sclerosis (Bragin et al. 2000). The effects of recurrent excitatory collateral synaptic connections on HFOs can be studied 
because synaptic connections between granule cells (GCs) do not exist in the healthy condition but are formed through mossy-fiber (GC axon) sprouting with sclerosis. As Bragin et al. (2002) performed electrical stimulations to evoke HFOs in the dentate gyrus in slice preparations, we simulated such experiments. In the present study, we could not model the actual physical properties of a macroelectrode, and therefore potentials were simply simulated as averaged fields in a measurement area that was far from neurons.

\section{METHODS}

In a real rat dentate gyrus that spans $6 \mathrm{~mm}$ in the septotemporal axis, there are more than one million neurons, and the dentate gyrus is divided into lamellae of $600 \mu \mathrm{m}$ each. The simulation of the rat dentate gyrus represented a three-dimensional structure of layers of neurons including 10,000 GCs and corresponding 300 mossy cells (MCs), 100 basket cells (BCs) and 120 hilar interneurons (HCs; hilar perforant-path associated cells, HIPP cells) with a spatial extent of $1 \mathrm{~mm}$ in the septotemporal and transverse axes. Somas of GCs were arranged to form the $60 \mu \mathrm{m}$-thick granule layer, and GCs projected dendrites into the molecular layer where they received the fibers from the perforant path. MCs were scattered in the polymorphic layer and BCs were along the border between the granule and polymorphic layers (Supplementary Figure S1A). In this simulation, GCs and MCs were excitatory with mediation by the a-amino-3-hydroxy-5-methylisoxazole-4-propionic acid (AMPA) receptor, and BCs and HCs were inhibitory through the fast $\gamma$-aminobutyric acid $(\mathrm{GABA})_{\mathrm{A}}$ receptor. The simulation program was a modified version of that used by Morgan and Soltesz (2008) with web data base based on a program of Dyhrfjeld-Johnson et al. (2007), and 
therefore gap junctions could not be included in the simulation. The relative number of cells and axonal distribution were in conformity to Morgan and Soltesz.

The single cells were conductance-based multi-compartmental models containing multiple ionic currents, including sodium, fast- and slow-delayed rectifier potassium, A-type potassium, $\mathrm{I}_{\mathrm{h}}, \mathrm{L}, \mathrm{N}$, and T-type calcium, and calcium-dependent potassium, with confirmation of firing patterns corresponding to recording from electrophysiological experiments (Santhakumar et al. 2005). The number of compartments in a model cell was $9,17,17$, and 13 , respectively regarding $\mathrm{GC}, \mathrm{MC}, \mathrm{BC}$, and $\mathrm{HC}$, and the number of dendrites in a cell was 2, 4, 4, and 4, respectively. Parameters of cells within each subgroup of $\mathrm{GC}, \mathrm{MC}, \mathrm{BC}$, and $\mathrm{HC}$ are common and not randomized. Mossy fiber sprouting was modeled by adding synaptic connections from GCs to the proximal dendrites of GCs. As in their simulation study, the probability of GC connection was modified for a preferential attachment, in which, at first, a half of the scheduled number of connections was randomly defined, and then the remaining connections were added so that the more connections a given cell already had, the more likely it would be to receive another connection (Barabási et al. 1999). The conduction delay including both the synaptic delay and the axonal conduction delay between GCs was at least $0.8 \mathrm{~ms}$, and the added distance-dependent delay was $0.24-\mathrm{ms}$ per $60-\mu \mathrm{m}$ separation. The degree of cell connectivity in the current simulations was in conformity to previous reports (Buckmaster et al. 2002; Dyhrfjeld-Johnson et al. 2007; Morgan and Soltesz 2008).

In a $10 \%$ stimulation paradigm, the perforant path synaptic input to the dentate gyrus was delivered to 1000 (10\%) GCs and the corresponding numbers of other cells in a $600 \mu \mathrm{m}$-wide lamina (Morgan and Soltesz 2008). The stimulations started $10 \mathrm{~ms}$ from the beginning of the session with temporal jitter within $2 \mathrm{~ms}$. We computed the current 
dipole moment (Demont-Guignard et al. 2009) generated by each GC and the field potentials recorded with microelectrodes (each $25 \mu \mathrm{m}$-by- $25 \mu \mathrm{m}$, an area of $625 \mu \mathrm{m}^{2}$ ) that were $1,000 \mu \mathrm{m}$ away from the border plane of molecular and granule layers in the direction of the molecular layer. The field generated by the other types of neurons was not computed because it is a closed field. Field potentials generated by every GC were summated over the electrodes under the quasi-static approximation. We assumed that the simulated field potentials corresponds to the signal induced by the network at the center of each microelectrode, that each GC functions as a point source (i.e., a current dipole formed by a sink and a source), and that all current dipoles are positioned within a volume conductor characterized by homogeneous conductivity (Supplementary

\section{Figure S1B).}

The potential $V_{i}$ of the electric dipole associated with a cell $i$ is

$$
V_{i}=\frac{\vec{d}_{i} \bullet \vec{u}_{i}}{4 \pi \sigma r_{i}^{2}}
$$

where $\sigma$ is the conductivity, $r_{i}$ is the distance from cell $i$ to the point of measurement (i.e. the recording electrode), $\vec{u}_{i}$ is a unit vector on the straight line going from cell $i$ to the recording point, and $\vec{d}_{i}$ is the dipole moment. With respect to the orientation, the dipole was aligned with the main axis of the cell, and the angle between this axis and the direction from the cell to the recording point was included in this dipole equation. We assumed that the sink and the source are located at the center of the somatic and dendritic compartments, respectively. The magnitude of current dipole moment $\vec{d}_{i}$ was computed for each GC as $\left\|\vec{d}_{i}\right\|=\left|V_{s}^{i}-V_{d}^{i}\right| g_{c} l_{i} S_{i}$, where $V_{s}^{i}$ and $V_{d}^{i}$, respectively, denote the somatic and dendritic membrane potentials, $g_{c}$ is the coupling conductance 
between the somatic and dendritic compartments, $l_{i}$ is the distance between the sink and the source (i.e. the pole separation), and $S_{i}$ is the total membrane surface. Regarding the numerical values, we used $\sigma=0.3 \mathrm{~S} / \mathrm{m}$ (Lindén et al. 2010), $g_{c}=1 \mathrm{mS} / \mathrm{cm}^{2}$ (adopted the value for hippocampal pyramidal cells) (Demont-Guignard et al. 2009), $l_{i}=258 \mu \mathrm{m}$ (the sum of half the size of the dendritic tree and the half of the soma size), and $S_{i}=10,749$ $\mu \mathrm{m}^{2}$ (calculated from the size of each compartment).

The measurement area (1 mm-by-1 mm, an area of $\left.1 \mathrm{~mm}^{2}\right)$ included 1600 (40-by-40) microelectrodes that were arranged parallel to the border plane. The field potentials recorded in this measurement area was approximated as the average potential of all the microelectrodes that were placed over the corresponding spatial extent; this process of simulation corresponded to the phenomenon of signal averaging. In the present setting, the recorded potential was supposed to be negative when excitatory PSP (EPSP) occurred in the GC dendrites, and positive when depolarization occurred in the GC somas. It has been reported that GCs generate a dipole field with an inward current (sink) in the molecular layer and an outward current (source) in the granule layer upon perforant path stimulation (Lomo et al. 1971; Bragin et al. 1995). The electrode potentials were referenced to zero.

In models of various degrees of sclerosis, the structural changes included loss of a percentage of MCs and $\mathrm{HCs}$ as well as the identical percentage of predetermined probability of connections between GCs (mossy fiber sprouting). The effect of density of overall cell connectivity was evaluated by arranging two sets of simulations with different probability of connectivity: in the relatively sparse and dense connectivity models with $100 \%$ sclerosis, sprouted mossy fibers contacted an average of $208 \pm 46$ and $411 \pm 88$ postsynaptic GCs, respectively. The effect of phasic GABAergic inhibition was 
tested by reducing it.

All simulations were performed in the NEURON version 7.2 environment (Carnevale and Hines 2006) (ModelDB database:

https://senselab.med.yale.edu/modeldb). Data analysis and plotting were done by using Matlab version 7.5.0 (Mathworks, USA). The sampling frequency of the simulated field potentials was $10 \mathrm{kHz}$.

\section{RESULTS}

\section{Generation of HFOs}

The observed evoked discharge in each model is depicted in the traces and the panels of corresponding time-frequency analysis by complex Morlet wavelet transform representing square-root power regarding the measurement area (Fig. 1 top row) and microelectrodes (Fig. 1, traces of a microelectrode at the center of the measurement area in middle row and that of a microelectrode at the border of granule and polymorphic layers in the dentate gyrus in bottom row). A discharge like an IED with a morphology comprising an initial positive deflection and a subsequent large negative deflection lasting approximately $50 \mathrm{~ms}$ was typically generated with associated HFOs superimposed over the ascending slope in the measurement area. The corresponding discharge recorded with a microelectrode at the center of the measurement area had a similar morphology and amplitude, because both the microelectrode and the whole measurement area were relatively far away from the cells, with an identical distance. The discharge recorded with a microelectrode in the dentate gyrus had a much higher amplitude and a reversed polarity of deflections (model with sparse cell connectivity and $100 \%$ sclerosis with full GABAergic inhibition shown in Fig. 1A,B). 
The increase in overall cell connectivity augmented discharges (Fig. 1C), and the loss of GABAergic inhibition that simulated the application of a $\mathrm{GABA}_{\mathrm{A}}$ blocker resulted in exaggeration of HFOs and reduction of the negative potentials (Fig. 1D), particularly in the model with dense connectivity of excitatory feedback between GCs and no inhibition (Fig. 1E). The peak frequency was $250 \mathrm{~Hz}$ in the model with $100 \%$ sclerosis, dense connectivity, and no inhibition.

In the models with no sclerosis, only the initial small discharge was evoked by the stimulation in case of full inhibition (Fig. 1F), and the loss of inhibition caused multiple bursts of activity with a peak frequency of $150 \mathrm{~Hz}$ (Fig. 1G). This activity may correspond to ripples recorded from the dentate gyrus with no sclerosis and the application of a GABA $A_{A}$ blocker (D'Antuono et al. 2005). These bursts of activity were assumed to be population spikes influenced by the input to GCs from MCs as there were no direct feedbacks between GCs.

\section{Relationship between cell firing and HFOs}

The relationship between the firing pattern of cells and the discharges may provide a cue to the process of generation of HFOs. In the sclerosis models (Fig. 2A-D), each positive peak in filtered traces (Fig. 2 red traces) largely coincided with a cluster of GC firing that repeated like ocean waves (Fig. 2 raster graphics; Fig. 2 C corresponding to Movie 1A) and with the corresponding peak in the histogram of GC firing pattern (Fig. 2 green traces, connected with arrows), each firing pattern having a high-frequency spectral blob (Fig. 2 spectra of firing histograms).

In the models with no sclerosis (excitatory input from MCs retained) and full GABAergic inhibition, an initial single cluster of GC firing (population spikes) had a 
faint spectral blob reflecting multiphasic morphology of the discharge (Fig. 2E).

Without inhibition, clusters of firing were observed as multiple pulses but did not form a pattern like ocean waves (Fig. 2F, corresponding to Movie 1B). The spectra of firing histogram showed the corresponding blob at approximately $150 \mathrm{~Hz}$ and spurious high-frequency blobs as well that did not correspond to actual firing. Thus it was shown that there may exist two different patterns of repetitive cell firing, and that the generation of truly oscillatory clustering of cell firing depends on the presence of excitatory feedback between GCs.

The relationship between membrane potentials, cell firing, and the generated discharges is illustrated in Fig. 3. Each positive peak of HFOs in filtered traces (top traces) largely corresponds to a peak in averaged soma membrane potentials that is actually averaged action potentials overriding depolarization (second row). The traces of soma membrane potentials of individual 100 representative GCs, each one sampled out of every 100 GCs (third row), illustrate the process of generation of action potentials triggered by stimulations through the perforant path (red circles). Depolarization of dendrite membrane potentials is also noted but lacks high-frequency components (fourth and bottom rows).

\section{Relationships between spectral power and frequencies of HFOs and the parameters of sclerosis}

To address the further question of how IEDs, particularly the associated HFOs, are generated, relationships were investigated between spectral power and frequencies of HFOs and each of the degree of sclerosis, cell connectivity, and inhibition. In the sclerosis models (Fig. 4 A,B), power of the spectral peaks of high-frequency blobs in 
time-frequency analysis increased in correspondence to progression of sclerosis, reduction of GABAergic inhibition, and increase in connectivity (top). The frequency of the spectral peaks of high-frequency blobs was highest in the $100 \%$ sclerosis model with no inhibition especially with dense cell connectivity. The relationships between spectral frequencies and the parameters were not completely consistent particularly in the models of mild sclerosis (bottom).

In the models with no sclerosis (Fig. 4C), the power of spectral blobs was high, but frequency represented the multiphasic morphology of individual pulses, particularly when a single pulse was evoked, and it did not show changes related to the degree of inhibition or connectivity, in contrast to the results in the sclerosis model.

\section{Factors influencing the results of simulations and robustness}

An increase of connectivity between GCs from 105 to 802 resulted in an increase of power and frequencies of HFOs (Fig. 5A,D, respectively). When the number of GCs stimulated by the perforant path increased from 100 to 6000, spectral power of HFOs accordingly increased (Fig. 5B), and spectral frequency of HFOs also slightly increased (Fig. 5E). When synaptic weight was increased, the power of HFOs slightly increased (Fig. 5C) and the frequency of HFOs increased up to $295 \mathrm{~Hz}$ (Fig. 5F).

Discharges simulated in a measurement area that was located $5 \mathrm{~mm}$ away from the model dentate gyrus were, compared to those at $1,000 \mu \mathrm{m}$, similar in morphology but much lower in amplitude (Fig. 6).

It may be suspected that HFOs were generated because the input from the perforant path was almost synchronous to trigger simultaneous initial firings. Actually an increase of temporal jitter in the perforant path stimulations up to $100 \mathrm{~ms}$ resulted in reduction of 
spectral power and frequency of HFOs, but HFOs were still generated (Fig. 7; Fig. 7B corresponding to Movie 1C).

The effects of noise were investigated by adding random values as white noise to dipole moment of each granule cell. The range of noise corresponded to membrane potentials of up to $50 \mathrm{mV}$. The field potentials of the microelectrode in the dentate gyrus include considerable noise, particularly when the added noise was large, but those of the measurement area and the microelectrode at the center of the measurement area are less noisy because of the summation of potentials contributed by a large number of cells with independent noise (Supplementary Figure S2).

\section{DISCUSSION}

In the current simulations of IEDs generated in the dentate gyrus with sclerosis, pathologic HFOs up to the fast ripple band occurred through the process of excitatory feedback between GCs, and such HFOs could be recorded in a remote measurement area of $1 \mathrm{~mm}^{2}$. Bragin et al. (2000) reported that "fast ripples were found in areas of dentate gyrus with more intensive mossy fiber sprouting", and stressed the importance of pathologically interconnected clusters of neurons in the generation of HFOs. Dzhala and Staley (2004) indicated that synchronous onset of action potential bursts mediated by recurrent excitatory synaptic transmission is important for the generation of fast ripples. Our findings confirmed such a hypothesis.

HFOs recorded with a microelectrode at the center of the measurement area were similar in morphology and amplitude to those recorded in the whole measurement area, showing that, when a microelectrode is far from the generator cells with an identical distance as the measurement area, recorded fields may be similar. As signal averaging 
occurs on the spatially extended area of measurement, results would be different depending on the location of electrode placement. HFOs recorded with a microelectrode close to cells had much higher amplitude in the current simulation, reflecting the known observation that the location of the microelectrode influences the recording of HFOs in the LFP. The spatial extent of the region generating the LFP is an important issue, and it is suggested the spatial reach of LFP may extend several millimeters (Kajikawa and Schroeder 2011; Lempka and McIntyre, 2013) and that it depends on the correlation of activity of generator cells (Lindén et al. 2011).

The emergence of HFOs are suggested to be caused by increased temporal scatter of spike-timing with decreased spatial synchronization (Foffani et al. 2007) and by out-of-phase firing (Ibarz et al. 2010). It was shown in the current simulation that firings can be partly synchronized (clustering of GC firings) and partly desynchronized (ocean wave-like repetition of out-of-phase firings) to allow the generation of HFOs recordable in the remote measurement area. A cluster of GC firing was associated with both HFOs and single population spikes, but their firing patterns were different: the firing pattern in a HFO was like ocean waves reflecting the underlying excitatory interconnections between GCs, in contrast to the pattern of explosive firings in single population spikes that did not depend on GC interconnections, although the two patterns may coexist in actual recordings. Note that HFOs were generated even when the input from the perforant path was asynchronous with a temporal jitter up to $100 \mathrm{~ms}$.

In the morphology of IEDs, soma depolarization associated with generation of action potentials and resulting HFOs appeared as the initial positivity, because soma depolarization was reflected in a positive potential, given the location of the measurement area. Very active generation of HFOs with positive deflections was 
associated with dissipation of negative deflections of IEDs, possibly because the current simulation involves a spatially limited part of dentate gyrus. As IEDs generally have a much wider spatial distributions than HFOs (Bragin et al. 2000), we speculate that the negative deflection of an IED may be generated through EPSPs of neurons distributed over an extended patch of tissue, and that only a small group of neurons actively fire with the generation of HFOs, and stimulate the surrounding neurons to cause EPSPs in them. Hence an epileptic neuronal network with a spatial extent wider than the current one $\left(1 \mathrm{~mm}^{2}\right)$ might generate greater slow negative potentials than those observed in the present study.

The spectral power and frequencies of HFOs appear to be influenced by various factors including the degree of sclerosis, cell connectivity and loss of GABAergic inhibition, as partly suggested previously (Stacey et al. 2009). The changes in overall cell connectivity might have complex effects which are possibly nonlinear. It was reported that the application of $\mathrm{GABA}_{\mathrm{A}}$ blockers augments HFOs (Bragin et al. 2002). In the present simulations, the observed HFOs had peak spectral frequencies $<300 \mathrm{~Hz}$ in time-frequency analysis, though spectral blobs had spectral components that spread up to $500 \mathrm{~Hz}$. Spectral frequencies of HFOs may be even higher if there were locally very dense connectivity and increase of synaptic weight at foci of HFO generation. The presence of gap junction might enhance HFOs because gap junctions are suggested to play an important role in HFOs (Traub and Biggig 2000; Hamzei-Sichani et al. 2007). Modification of the properties of ion channel mechanisms and addition of synaptic noise may also influence HFOs but they are beyond the scope of the present study. The involvement of N-methyl-D-aspartate (NMDA) receptors in the generation of HFOs is suggested and should be studied further (Moschovos et al. 2008). We hope to perform 
more elaborate and realistic simulations including gap junctions, NMDA receptors, and many other factors in the future.

The usage of the current dipole model with line source approximation might influence the results in this study, but the dipole model is generally valid in EEG (Einevoll et al. 2013). The measurement area was located 1,000 $\mu \mathrm{m}$ away from the dentate gyrus, and the dipole model gives a good approximation of electric field when the distance between the electrode and the current generator (neurons) is three or four times the pole separation, which was $258 \mu \mathrm{m}$ for the GCs of the current study (Nunez and Srinivasan 2006). Potentials in a measurement area $5 \mathrm{~mm}$ away from the dentate gyrus showed discharges that were quite similar though low in amplitude, thus reinforcing the validity of the dipole model. It is also of note that HFOs associated with action potentials could be recorded with this remote electrode, in contrast to a belief that fields generated by action potentials are visible only at very short distances from the generator (Bédard et al. 2006). However the additional simulation of LFP recorded with a microelectrode close to cells in the dentate gyrus was theoretically imprecise, though it was necessary for comparison. The amplitude of IEDs and HFOs may be somewhat different depending on parameters of model cells that influence the magnitude of the current dipole moment. It was difficult for us to perform the simulation of an actual macroelectrode with uniform potential imposed over its large surface and the effects of such an object on the neuronal network. Therefore averaged potentials of microelectrodes in the measurement area of the current study could not be regarded as an exact model of macroelectrode. If we could make a first approximation of a macroelectrode in a way including average of microelectrode potentials, then it may be possible to see HFOs on such a simplistic macroelectrode. The effects of experimental 
noise and noise from other neural sources are also important factors, but the systematic evaluation of such effects would require another series of extensive simulations. These questions need to be studied or simulated in future studies.

We are aware that any simulation is a simplistic model of the actual neuronal tissue. Although we have 10,000 GCs and three other types of cells in the patch of model dentate gyrus, there are more than a million neurons in the real rat dentate gyrus (Morgan and Soltesz 2008). Factors of hippocampal sclerosis other than loss of interneurons and mossy-fiber sprouting could not be included in the model. We, however, could illustrate through the current simulations that action potentials can be clustered to generate an electrical field which contributes to the electrical field recorded not only by microelectrodes but also in a measurement area that is far from neurons: this field fluctuates in the form of HFOs. If the net effects of MCs are not excitatory but rather inhibitory, as suggested by Jinde et al. (2012), a different simulation model should be constructed, but the models with no inhibition would remain similar. The generation of physiologic high-frequencies is another important issue. The correspondence between simulations and real experiments using brain slices of the animal models of sclerosis and post-operative human tissue should be further studied. Simulations and real experiments would complement each other to allow deeper insight to the generation process of IEDs and HFOs. As this study was the first to consider action potentials as contributors to field potentials far from neurons, EEG probably harbors information about neuronal networks that has yet to be recognized. We hope that additional new meaning could extend the clinical utility of depth EEG beyond its traditional usage. 


\section{REFERENCES}

Barabási AL, Albert R. Emergence of scaling in random networks. Science 1999;286:509-512.

Bédard C, Kröger H, Destexhe A. Model of low-pass filtering of local field potentials in brain tissue. Phys Rev E Stat Nonlin Soft Matter Phys 2006;73:051911.

Bragin A, Jandó G, Nádasdy Z, Hetke J, Wise K, Buzsáki G. Gamma (40 - 100 Hz) oscillation in the hippocampus of the behaving rat. J Neurosci 1995;15:47-60.

Bragin A, Engel Jr J, Wilson CL, Fried I, Mathern GW. Hippocampal and entorhinal cortex high-frequency oscillations $(100-500 \mathrm{~Hz})$ in human epileptic brain and in kainic acid-treated rats with chronic seizures. Epilepsia 1999;40:127-137

Bragin A, Wilson CL, Engel J Jr. Chronic epileptogenesis requires development of a network of pathologically interconnected neuron clusters: a hypothesis. Epilepsia 2000;41 (Suppl 6):S144-S152.

Bragin A, Mody I, Wilson CL, Engel J Jr. Local generation of fast ripples in epileptic brain. J Neurosci 2002;22:2012-2021.

Bragin A, Benassi SK, Kheiri F, Engel J Jr. Further evidence that pathologic high-frequency oscillations are bursts of population spikes derived from recordings of identified cells in dentate gyrus. Epilepsia 2011;52:45-52.

Buckmaster PS, Zhang GF, Yamawaki R. Axon sprouting in a model of temporal lobe epilepsy creates a predominantly excitatory feedback circuit. J Neurosci 2002;22:6650-6658.

Buzsáki G, Anastassiou CA, Koch C. The origin of extracellular fields and currents--EEG, ECoG, LFP and spikes. Nat Rev Neurosci 2012;13:407-420.

Carnevale NT, Hines ML. The Neuron Book. UK: Cambridge University Press, 2006. 
Châtillon CÉ, Zelmann R, Bortel A, Avoli M, Gotman J. Contact size does not affect high frequency oscillation detection in intracerebral EEG recordings in a rat epilepsy model. Clin Neurophysiol 2011;122:1701-1705.

Châtillon CE, Zelmann R, Hall JA, Olivier A, Dubeau F, Gotman J. Influence of contact size on the detection of HFOs in human intracerebral EEG recordings. Clin Neurophysiol 2013;124:1541-1546.

D'Antuono M, de Guzman P, Kano T, Avoli M. Ripple activity in the dentate gyrus of dishinibited hippocampus-entorhinal cortex slices. J Neurosci Res 2005;80:92-103.

Demont-Guignard S, Benquet P, Gerber U, Wendling F. Analysis of intracerebral EEG recordings of epileptic spikes: insights from a neural network model. IEEE Trans Biomed Eng 2009;56:2782-2795.

Demont-Guignard S, Benquet P, Gerber U, Biraben A, Martin B, Wendling F. Distinct hyperexcitability mechanisms underlie fast ripples and epileptic spikes. Ann Neurol 2012;71:342-352.

Dyhrfjeld-Johnson J, Santhakumar V, Morgan RJ, Huerta R, Tsimring L, Soltesz I. Topological determinants of epileptogenesis in large-scale structural and functional models of the dentate gyrus derived from experimental data. J Neurophysiol 2007;97:1566-1587.

Dzhala VI, Staley KJ. Mechanisms of fast ripples in the hippocampus. $J$ Neurosci 2004;24:8896-8906.

Einevoll GT, Kayser C, Logothetis NK, Panzeri S. Modelling and analysis of local field potentials for studying the function of cortical circuits. Nat Rev Neurosci 2013;14:770-785.

Engel J Jr, Bragin A, Staba R, Mody I. High-frequency oscillations: what is normal and 
what is not? Epilepsia 2009;50:598-604.

Foffani G, Uzcategui YG, Gal B, Menendez de la Prida L. Reduced spike-timing reliability correlates with the emergence of fast ripples in the rat epileptic hippocampus. Neuron 2007;55:930-941.

Gold C, Henze DA, Koch C, Buzsáki G. On the origin of the extracellular action potential waveform: A modeling study. J Neurophysiol 2006);95:3113-3128.

Hamzei-Sichani F, Kamasawa N, Janssen WG, Yasumura T, Davidson KG, Hof PR, et al. Gap junctions on hippocampal mossy fiber axons demonstrated by thin-section electron microscopy and freeze fracture replica immunogold labeling. Proc Natl Acad Sci USA 2007;104:12548-12553.

Ibarz JM, Foffani G, Cid E, Inostroza M, Menendez de la Prida L. Emergent dynamics of fast ripples in the epileptic hippocampus. J Neurosci 2010;30:16249-16261.

Jacobs J, Zijlmans M, Zelmann R, Chatillon CE, Hall J, Olivier A, et al. High-frequency electroencephalographic oscillations correlate with outcome of epilepsy surgery. Ann Neurol 2010;67:209-220.

Jinde S, Zsiros V, Jiang Z, Nakao K, Pickel J, Kohno K, et al. Hilar mossy cell degeneration causes transient dentate granule cell hyperexcitability and impaired pattern separation. Neuron 2012;76:1189-200.

Jirsch JD, Urrestarazu E, LeVan P, Olivier A, Dubeau F, Gotman J. High-frequency oscillations during human focal seizures. Brain 2006;129: 1593-1608.

Kajikawa Y, Schroeder CE. How local is the local field potential? Neuron 2011;72:847-858.

Lempka SF, McIntyre CC. Theoretical analysis of the local field potential in deep brain stimulation applications. PLoS One. 2013;8:e5983 
Lindén H, Pettersen KH, Einevoll GT. Intrinsic dendritic filtering gives low-pass power spectra of local field potentials. J Comput Neurosci 2010;29:423-444.

Lindén H, Tetzlaff T, Potjans TC, Pettersen KH, Grün S, Diesmann M, et al. Modeling the spatial reach of the LFP. Neuron 2011;72:859-872.

Lomo T. Patterns of activation in a monosynaptic cortical pathway: the perforant path input to the dentate area of the hippocampal formation. Exp Brain Res 1971;12:18-45.

Menendez de la Prida L, Trevelyan AJ. Cellular mechanisms of high frequency oscillations in epilepsy: on the diverse sources of pathological activities. Epilepsy Res 2011;97:308-317.

Morgan RJ, Soltesz I. Nonrandom connectivity of the epileptic dentate gyrus predicts a major role for neuronal hubs in seizures. Proc Natl Acad Sci USA 2008;105:6179-6184.

Moschovos C, Kostopoulos G, Papatheodoropoulos C. Long-term potentiation of high-frequency oscillations and synaptic transmission characterize in vitro NMDA receptor-dependent epileptogenesis in the hippocampus. Neurobiol Dis 2008;29:368-380.

Nunez PL, Srinivasan R. Electric Fields of the Brain: The Neurophysics of EEG, 2nd Edition. UK: Oxford University Press, 2006.

Reimann MW, Anastassiou CA, Perin R, Hill SL, Markram H, Koch C. A biophysically detailed model of neocortical local field potentials predicts the critical role of active membrane currents. Neuron 2013;79:375-390.

Santhakumar V, Aradi I, Soltesz I. Role of mossy fiber sprouting and mossy cell loss in hyperexcitability: a network model of the dentate gyrus incorporating cell types and 
axonal topography. J Neurophysiol 2005;93:437-453.

Schomburg EW, Anastassiou CA, Buzsáki G, Koch C. The spiking component of oscillatory extracellular potentials in the rat hippocampus. J Neurosci 2012;32:11798-11811.

Stacey WC, Lazarewicz MT, Litt B. Synaptic noise and physiological coupling generate high-frequency oscillations in a hippocampal computational model. J Neurophysiol 2009;102:2342-2357.

Traub RD, Bibbig A. A model of high-frequency ripples in the hippocampus based on synaptic coupling plus axon-axon gap junctions between pyramidal neurons. $J$ Neurosci 2000;20:2086-2093.

Wendling F, Bartolomei F, Mina F, Huneau C, Benquet P. Interictal spikes, fast ripples and seizures in partial epilepsies--combining multi-level computational models with experimental data. Eur J Neurosci 2012;36:2164-2177. 


\section{FIGURE LEGENDS}

\section{Figure 1. Generated discharges in the model dentate gyrus}

Traces of field potentials and panels of the corresponding spectral analysis are depicted with respect to recording with the measurement area (top row), a microelectrode at the center of the measurement area (middle row above the separator line), and a microelectrode at the border of granule and polymorphic layers in the dentate gyrus (bottom row). Traces with no filter are shown in green, and traces low-cut filtered (-6 $\mathrm{dB})$ at $80 \mathrm{~Hz}$ and $250 \mathrm{~Hz}$ shown in blue and red, respectively. The traces are arranged with upward negativity as in case of clinical EEG. In the model with $100 \%$ sclerosis, sparse cell connectivity and full GABAergic inhibition, HFOs with spectral peak frequency of $190 \mathrm{~Hz}$ are observed superimposed on the beginning of a slower deflection of the IED that was negative with the measurement area and positive with the microelectrode in the dentate gyrus (magnified traces in $\mathbf{A}$; overlaid traces in $\mathbf{B}$ ). In the model with $100 \%$ sclerosis, dense connectivity and full inhibition, HFOs with peak frequency of $230 \mathrm{~Hz}$ are found $(\mathbf{C})$. In the models with $100 \%$ sclerosis and no inhibition, HFOs with peak frequencies of $230 \mathrm{~Hz}$ and $250 \mathrm{~Hz}$ are observed with sparse (D) and dense (E) connectivity, respectively. In models with no sclerosis and dense connectivity, only the initial small discharge was evoked in case of full inhibition $(\mathbf{F})$, and a burst of activity was observed with spectral frequency of $150 \mathrm{~Hz}$ in case of no inhibition $(\mathbf{G})$. Traces generally had similar morphology and amplitude in the recording with a microelectrode as with the measurement area when the microelectrode was at the center of the measurement area because both the microelectrode and the measurement area were relatively far away from the generator cells, with an identical distance. But traces 
have more complex morphology and much higher amplitude in the recording with a microelectrode in the dentate gyrus. LCF: low-cut frequency.

\section{Figure 2. Relationships between the cell firing pattern and discharges}

Each positive peak in traces filtered at $250 \mathrm{~Hz}$ (red traces) largely coincides with a burst of granule cell (GC) firing (raster graphics) and the corresponding peak in the histogram of GC firing pattern (green traces; connected with arrows), each firing pattern having a spectral blob (spectra of histograms of GC firing pattern). Columns A-D represent models with sclerosis: (A) model with sparse cell connectivity and full GABAergic inhibition, (B) model with dense connectivity and full inhibition, (C) model with sparse connectivity and no inhibition, and (D) model with dense connectivity and no inhibition (corresponding to Fig. 1B-E, respectively). Columns $\mathbf{E}$ and $\mathbf{F}$ represent models with no sclerosis (full inhibition in $\mathbf{E}$ and no inhibition in $\mathbf{F}$, corresponding to Fig. 1F,G, respectively). The raster graphics of cell firing in $\mathbf{C}$ and $\mathbf{F}$ correspond to Movie 1A and B, respectively. MCs, mossy cells; BCs, basket cells; HCs, hilar perforant-path associated cells.

Figure 3. Relationships between membrane potentials with action potentials and HFOs

Columns A: sclerosis model with sparse connectivity and no GABAergic inhibition; B: sclerosis model with dense connectivity and no inhibition (corresponding to Fig. 1D,E, respectively). Each positive peak in traces filtered at $250 \mathrm{~Hz}$ (top red traces) largely corresponds to averaged soma membrane potentials of firings (green traces in second row; connected with arrows). The traces of soma membrane potentials of individual 100 
representative GCs, each one sampled out of every 100 GCs, are depicted below (third row) with stimulations by the perforant path (red circles). This transient soma membrane depolarization with superimposed action potentials may correspond to the paroxysmal depolarization shift (PDS). Depolarization of dendrite membrane potentials is also observed but lacks high-frequency components (forth row: averaged potentials; bottom row: potentials of the sampled $100 \mathrm{GCs}$ ).

Figure 4. Relationships between spectral power and frequencies of HFOs and each of the degree of sclerosis, cell connectivity, and inhibition

Changes of spectral power are depicted in the top row and changes of frequencies in the bottom row. In the sclerosis models, spectral power of generated HFOs consistently increased with the progression of sclerosis from $25 \%$ to $100 \%$, and with the reduction of GABAergic inhibition from $100 \%$ to $0 \%$, in the model of relatively sparse (A) and dense connectivity (B). Spectral frequency of HFOs was highest with 100\% sclerosis and no inhibition in each model of sparse and dense cell connectivity, though the relationships between spectral frequencies and the parameters were not completely consistent particularly in the models of mild sclerosis. In the models with no sclerosis (C), spectral power was high, and spectral frequency was especially low with no inhibition.

\section{Figure 5. Factors influencing the results of simulations}

An increase of the degree of GC connectivity (average number of GC connections, 1: $105 \pm 24,2: 208 \pm 46,3: 411 \pm 88,4: 609 \pm 128,5: 802 \pm 166)$ was associated with an increase of power and frequencies of HFOs, as indicated in $\mathbf{A}$ and $\mathbf{D}$, respectively. An 
increase of the number of GCs stimulated by the perforant path was related to an increase of power of HFOs (B) and with a slight increase of frequency of HFOs (E). An increase of synaptic weight also had an effect to increase HFO power (C) and frequencies of HFOs up to $295 \mathrm{~Hz}(\mathbf{F})$.

Figure 6. Generated discharges recorded in a measurement area $5 \mathrm{~mm}$ away from the model dentate gyrus

The traces (top row) and the panels of corresponding time-frequency analysis (bottom row) depicts discharges recorded in a measurement area that was similar to one used in Fig. 1 but $5 \mathrm{~mm}$ away from the dentate gyrus. The discharges recorded at $5 \mathrm{~mm}$ were, compared to those at $1,000 \mu \mathrm{m}$, similar in morphology though low in amplitude.

Columns A-F correspond to Fig. 1 B-G respectively.

\section{Figure 7. Effects of jitter in stimulation}

In the models with dense connectivity and $100 \%$ sclerosis, the temporal jitter in the perforant path stimulation was increased to $100 \mathrm{~ms}$, and HFOs were still observed though spectral power and frequency of the generated HFOs decreased (A, full inhibition; B, no inhibition corresponding to Movie 1C). 


\section{Figures}

\section{Figure 1}

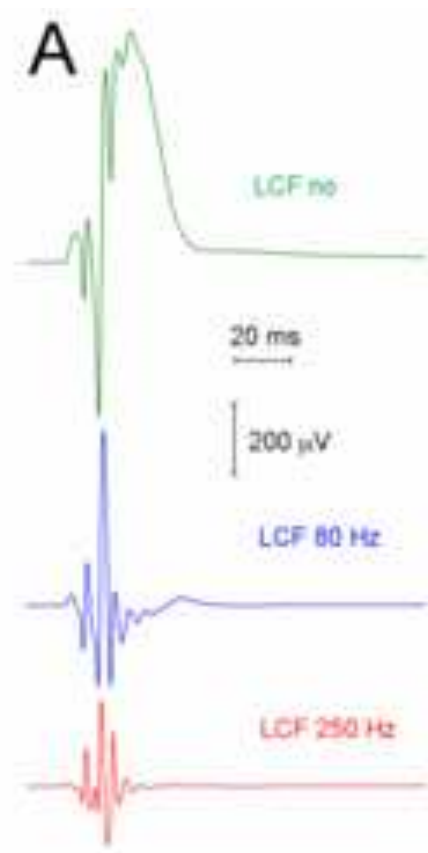

B $\quad$ C

Measurement area

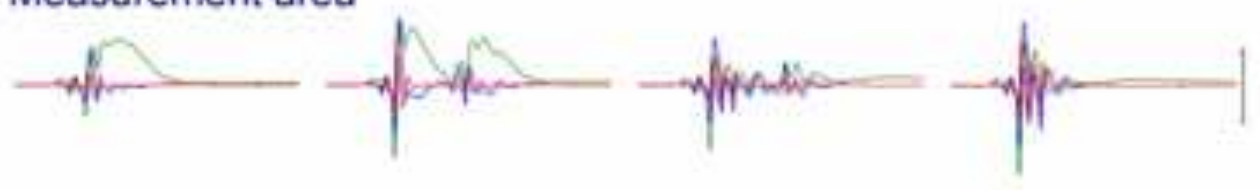

$\mathrm{F}$

G
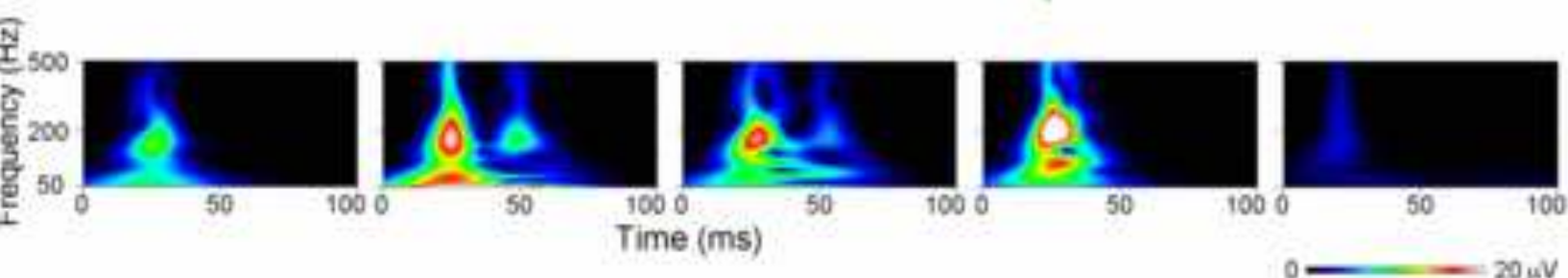

50 $20,4 \mathrm{~V}$
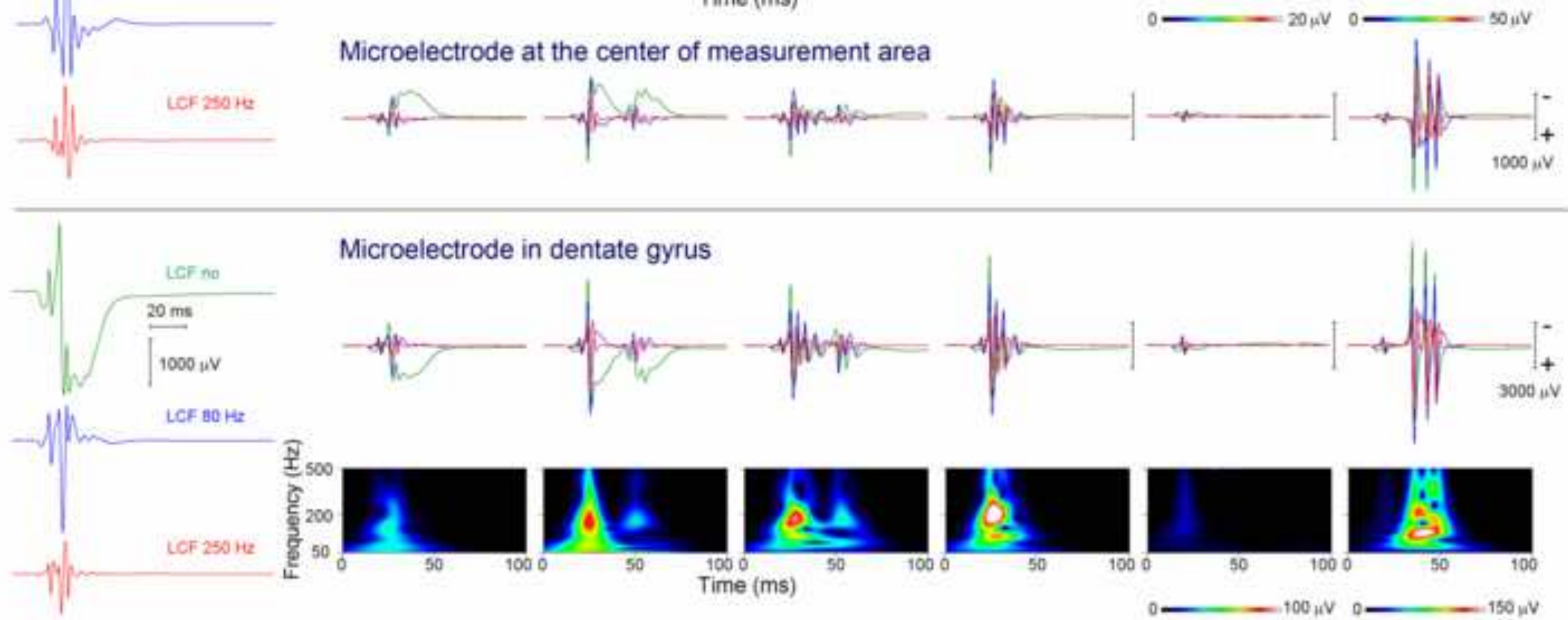

Microelectrode at the center of measurement area
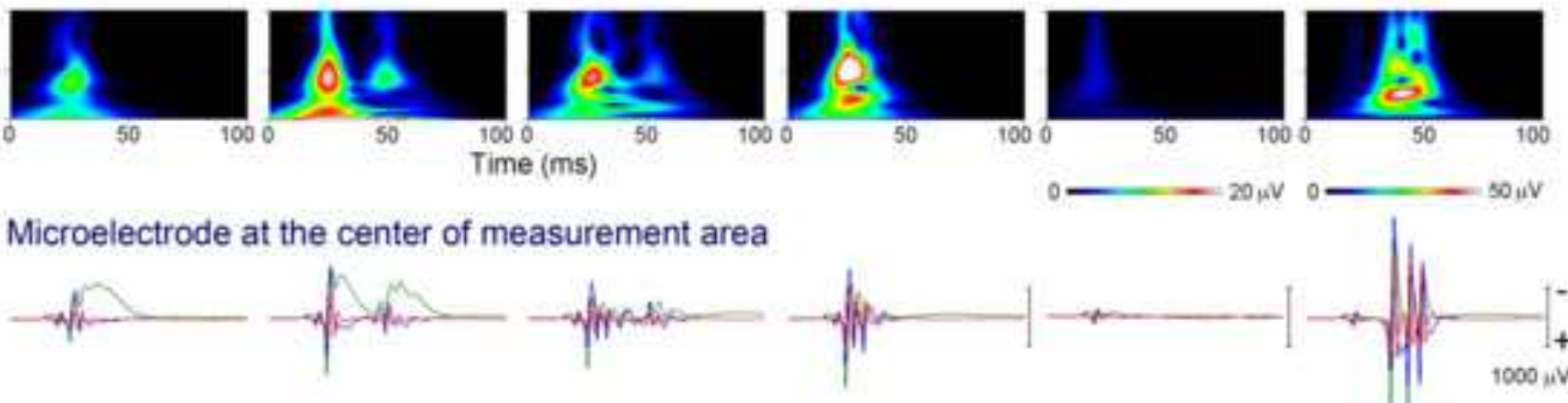

Microelectrode in dentate gyrus
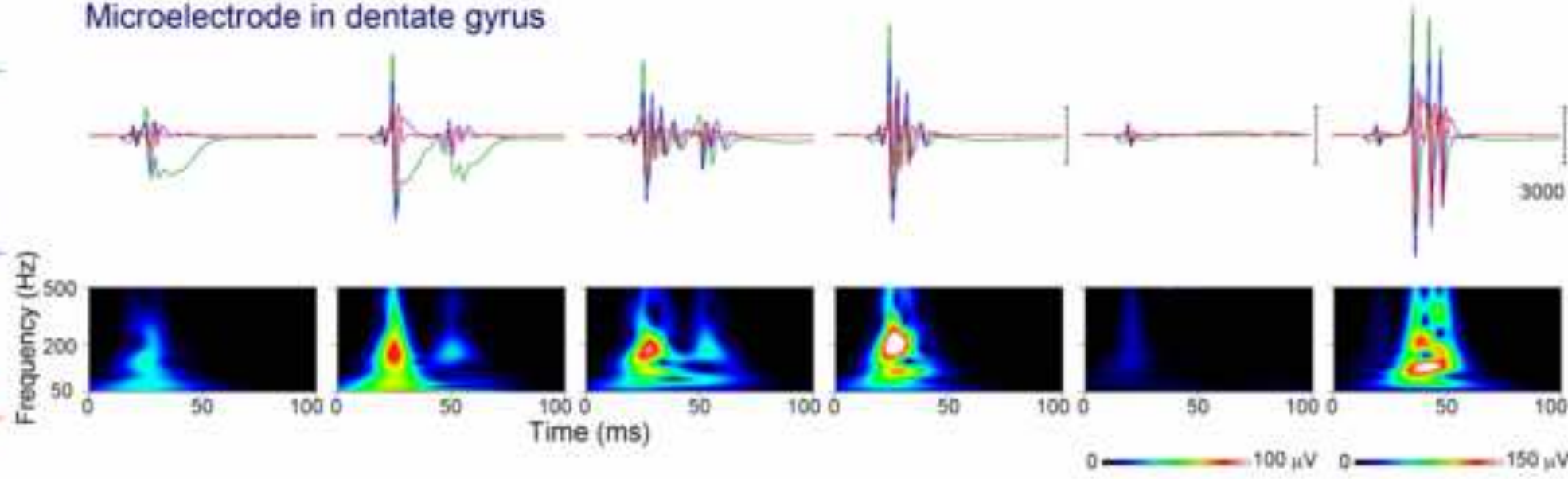

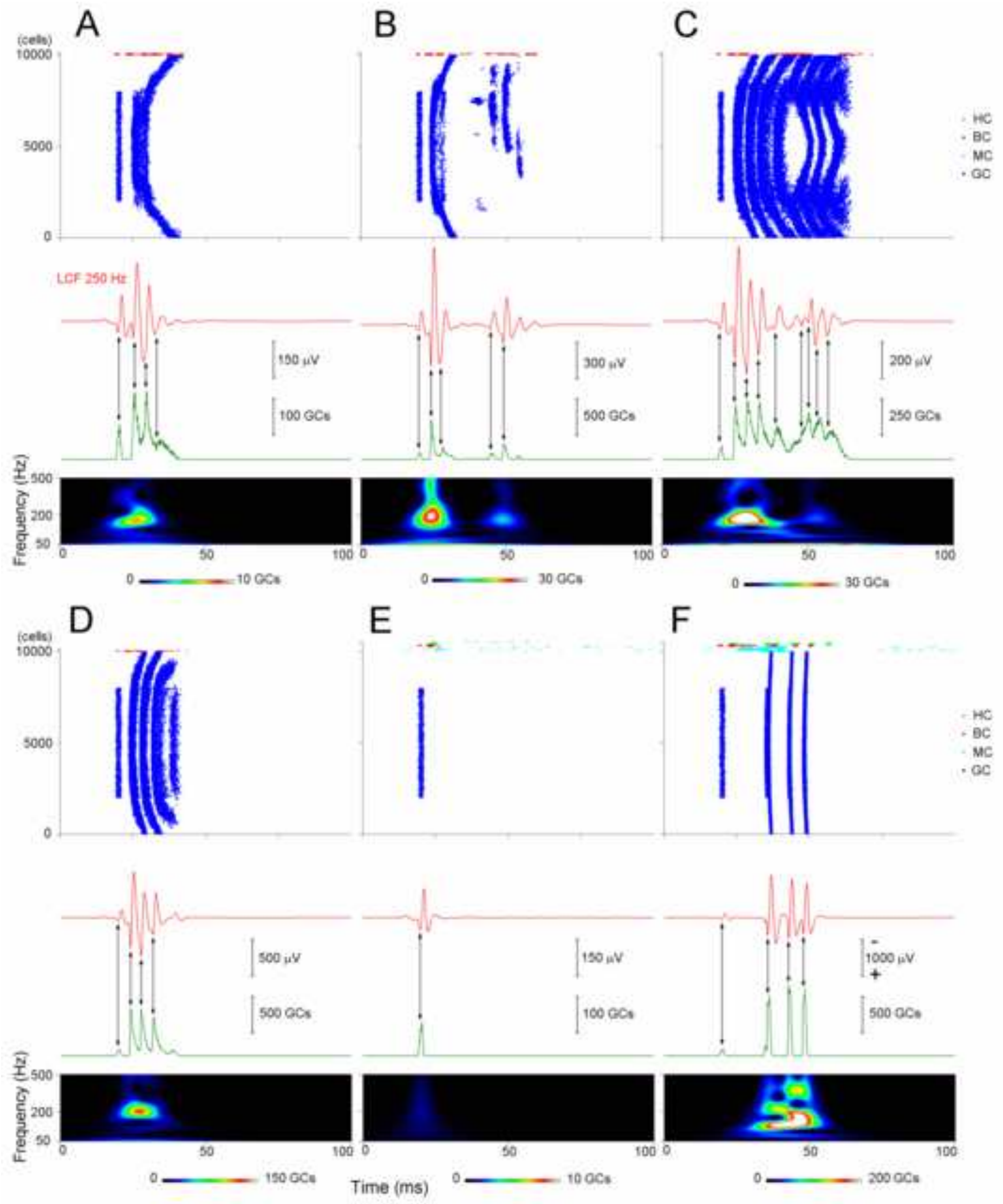

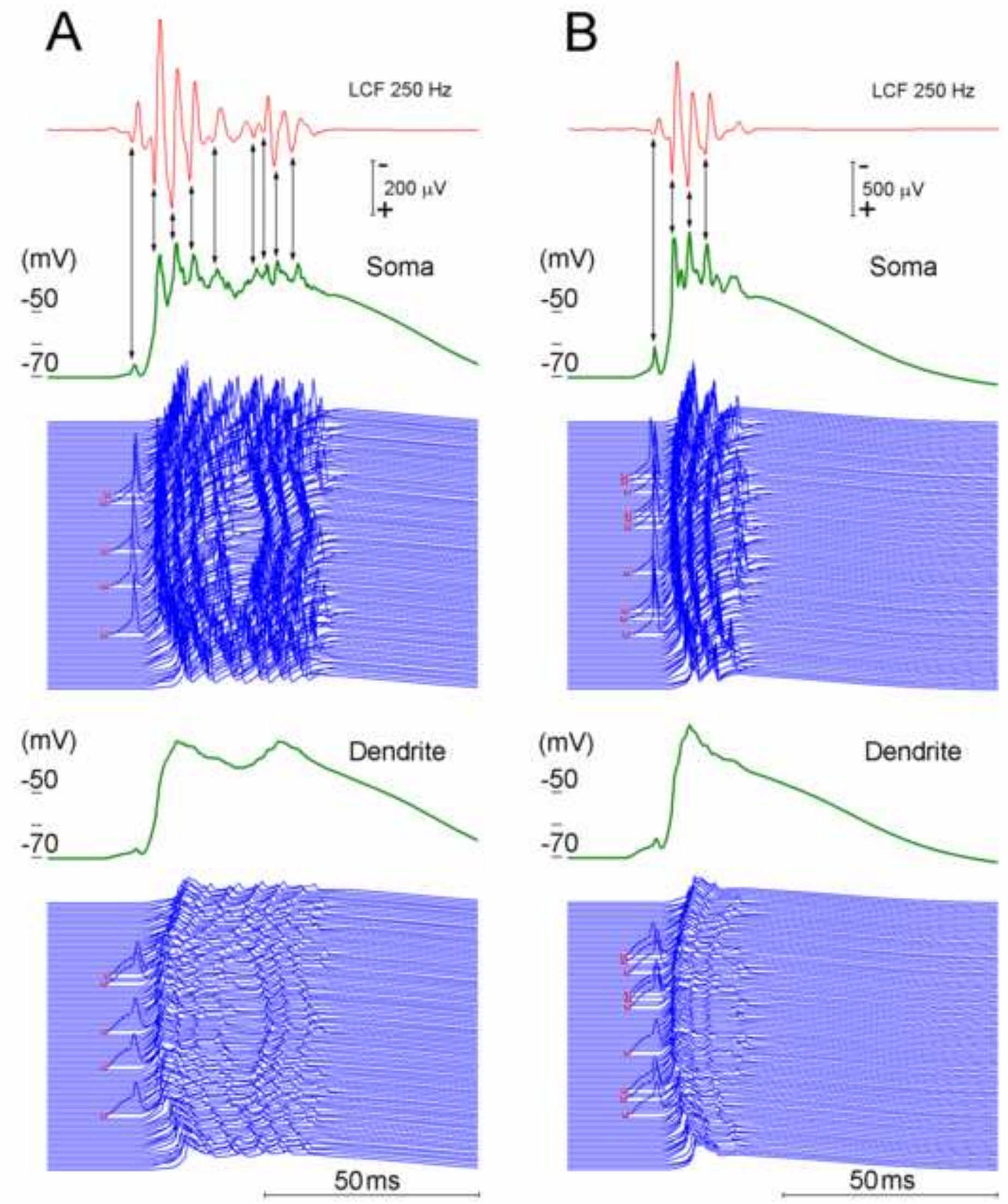

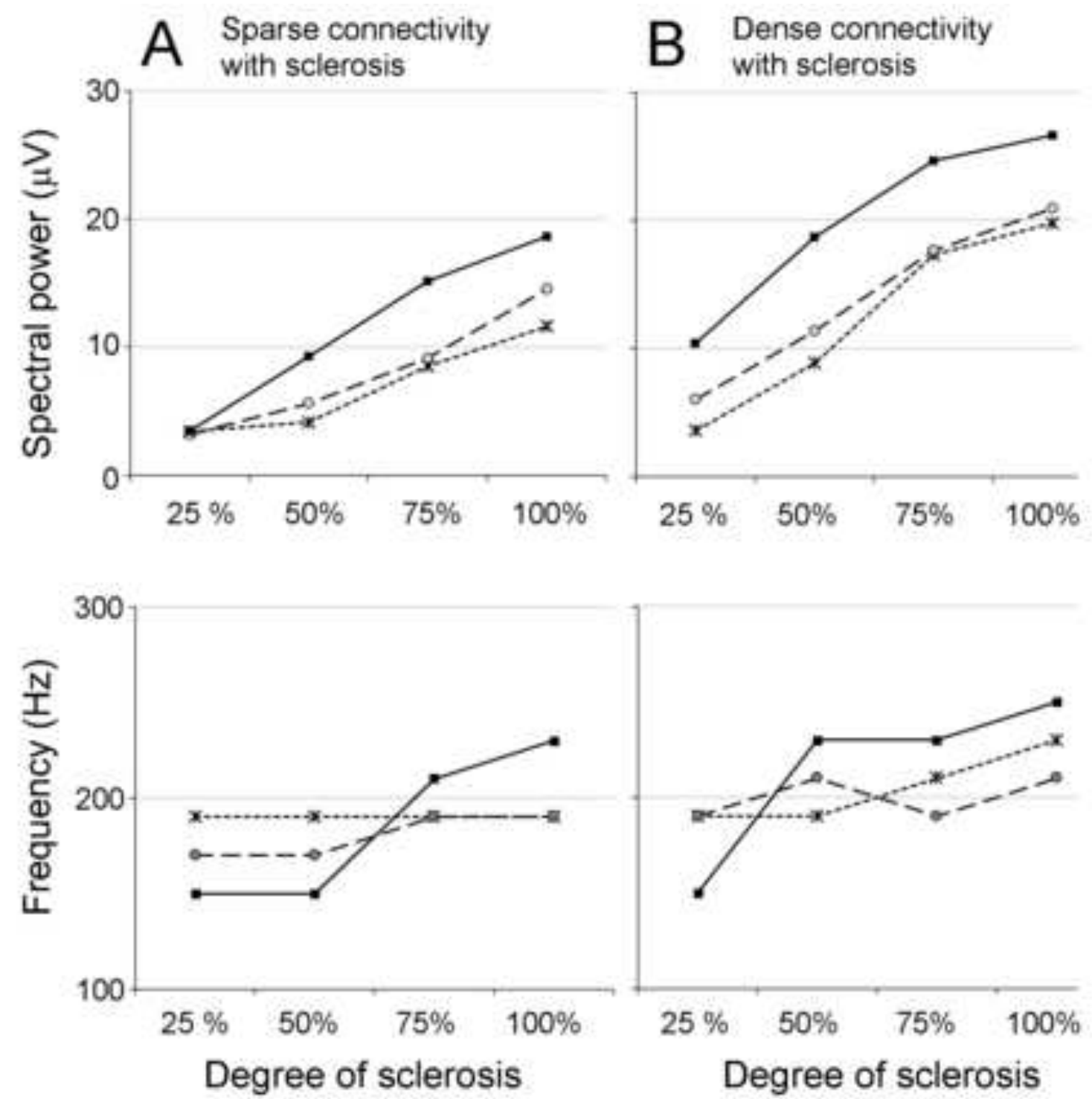
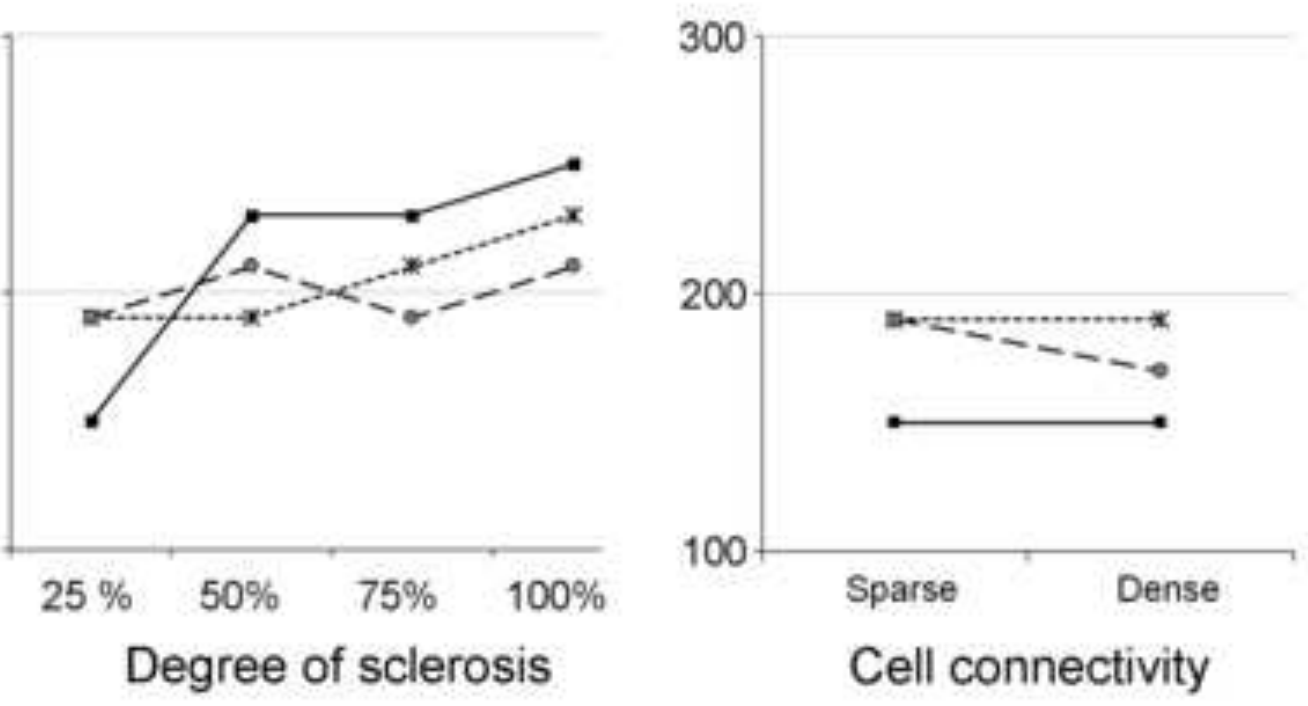

Cell connectivity
- full inhibition

- $50 \%$ inhibition

- no inhibition

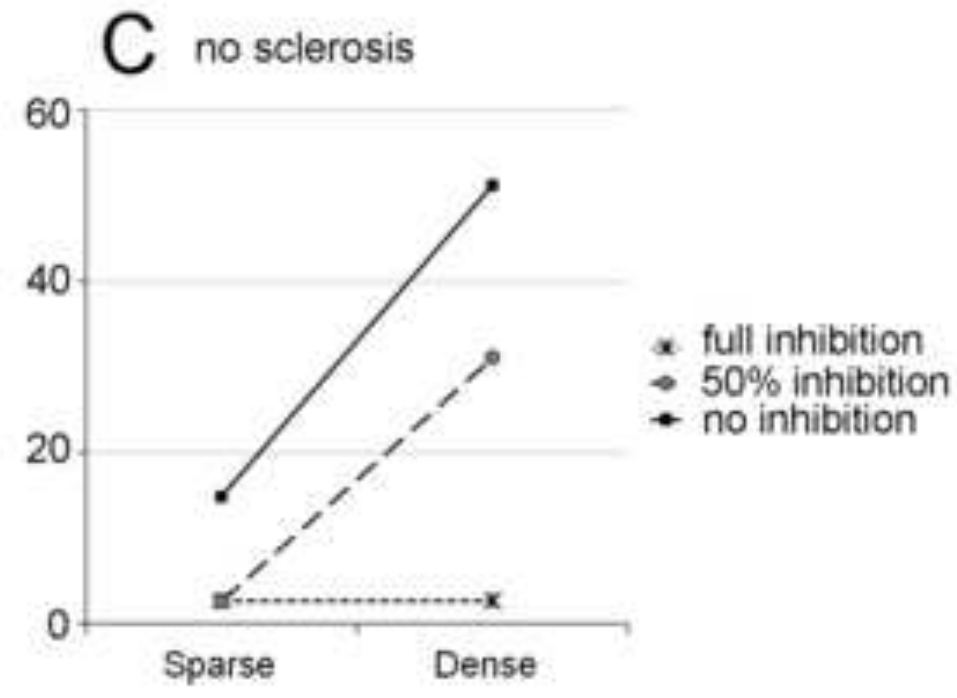



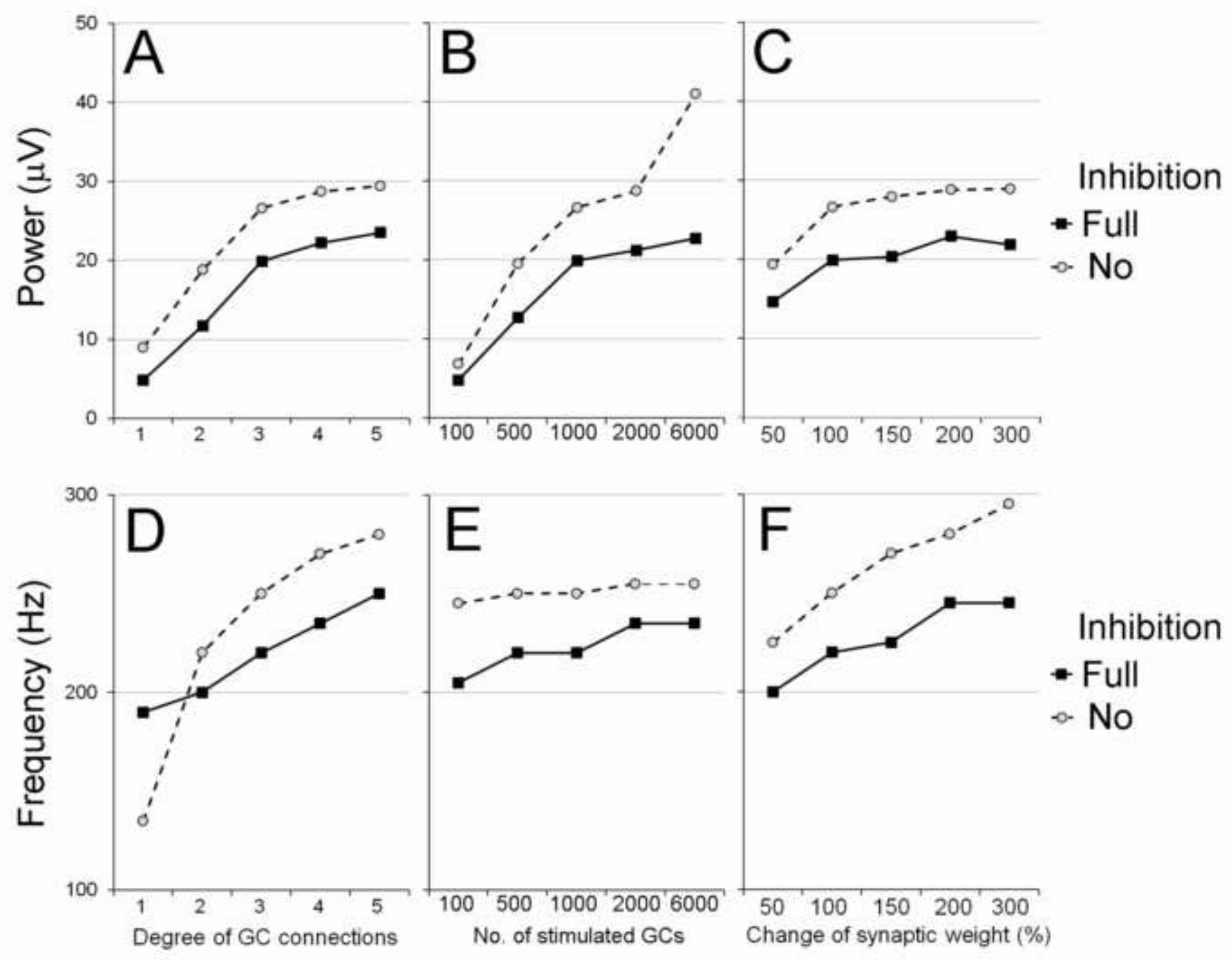

Inhibition

- Full

- No

Fure

No. of stimulated GCs

Change of synaptic weight $(\%)$ 

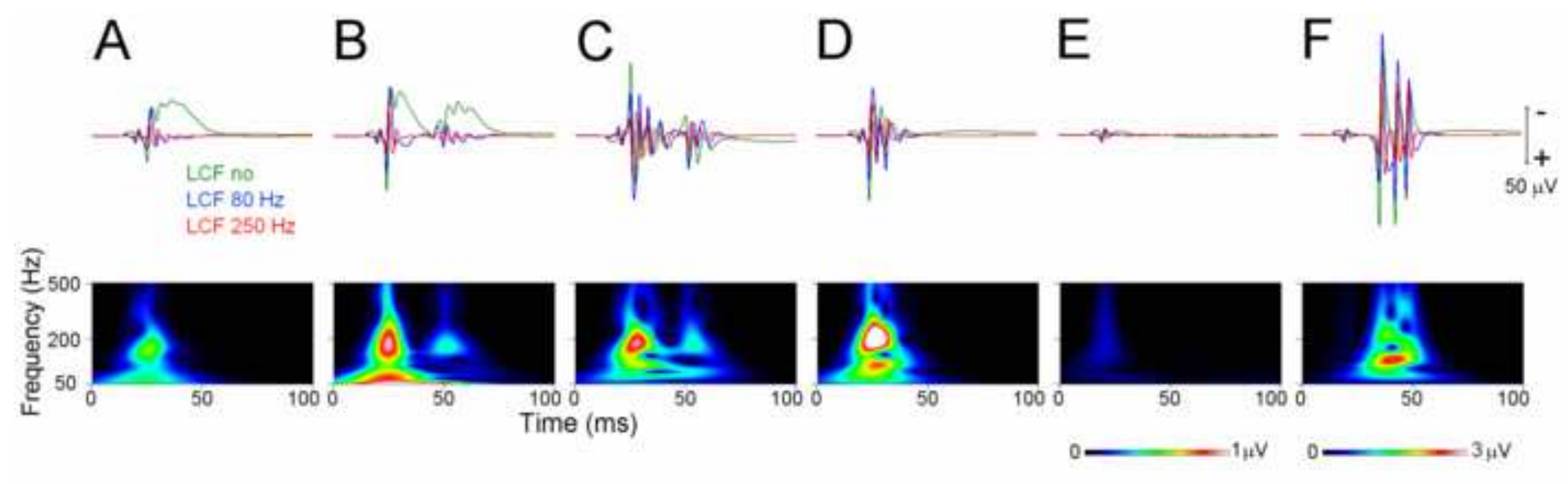

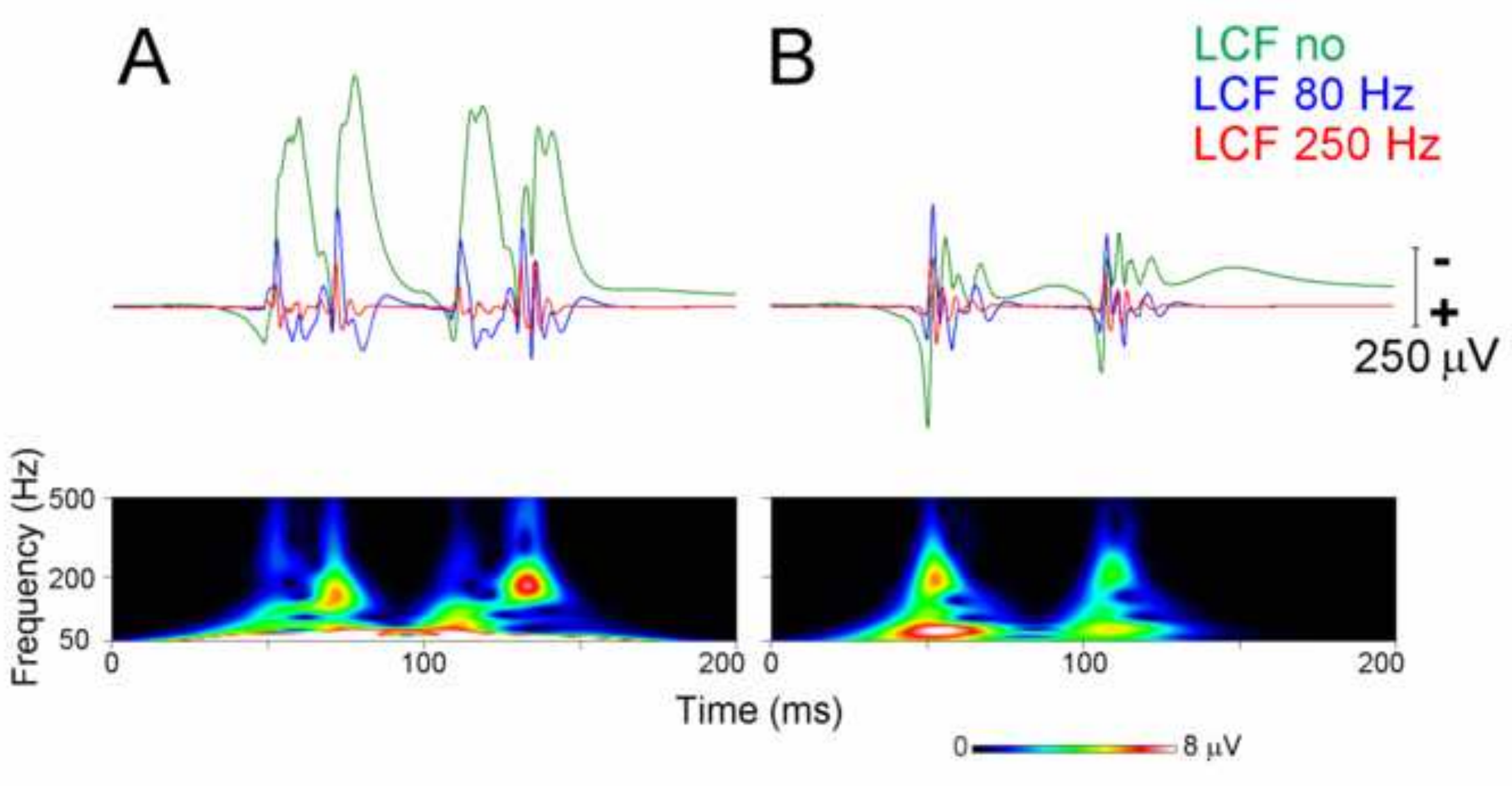

LCF no LCF $80 \mathrm{~Hz}$ LCF $250 \mathrm{~Hz}$

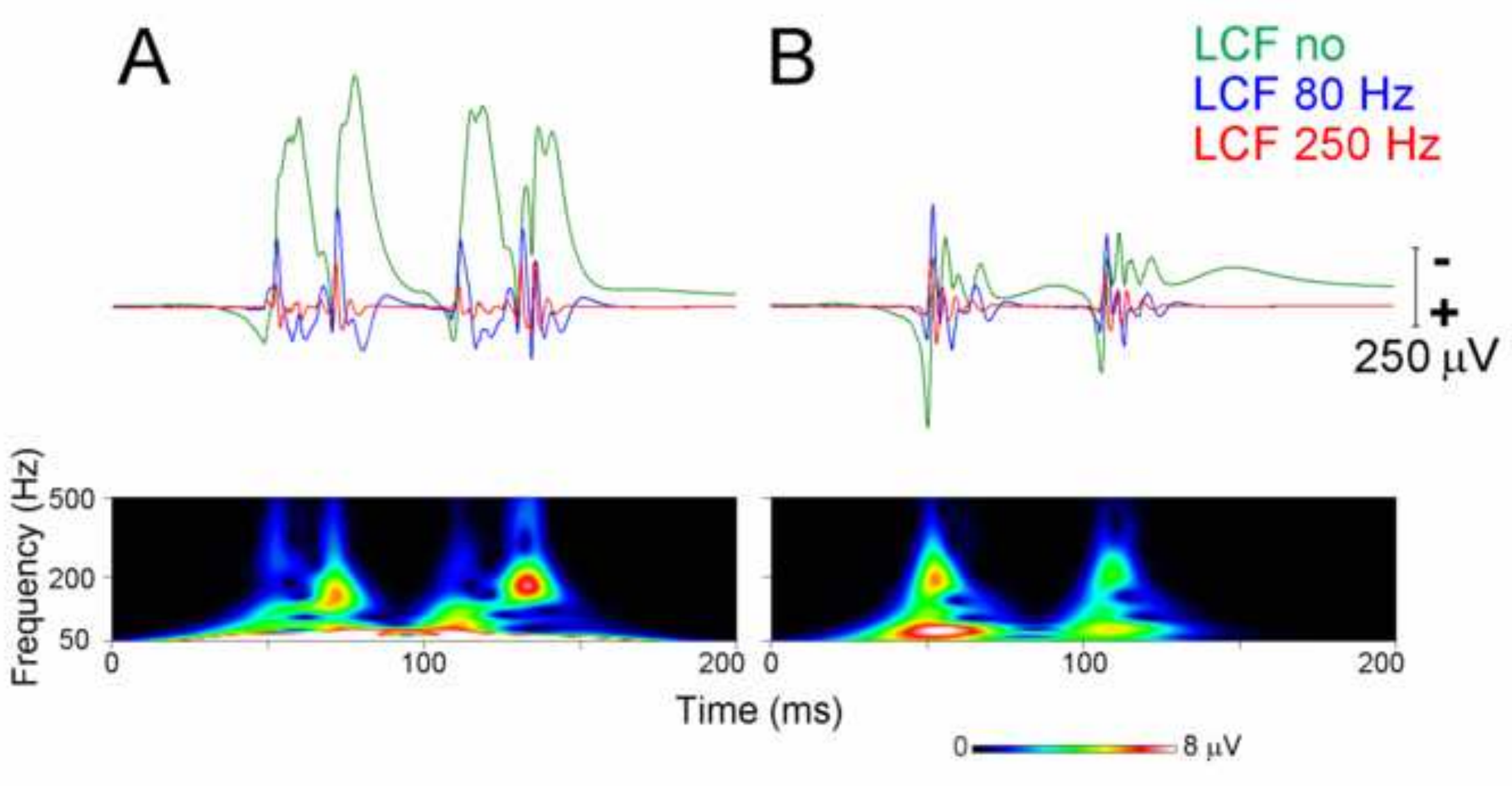

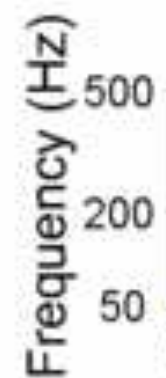

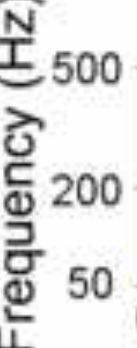

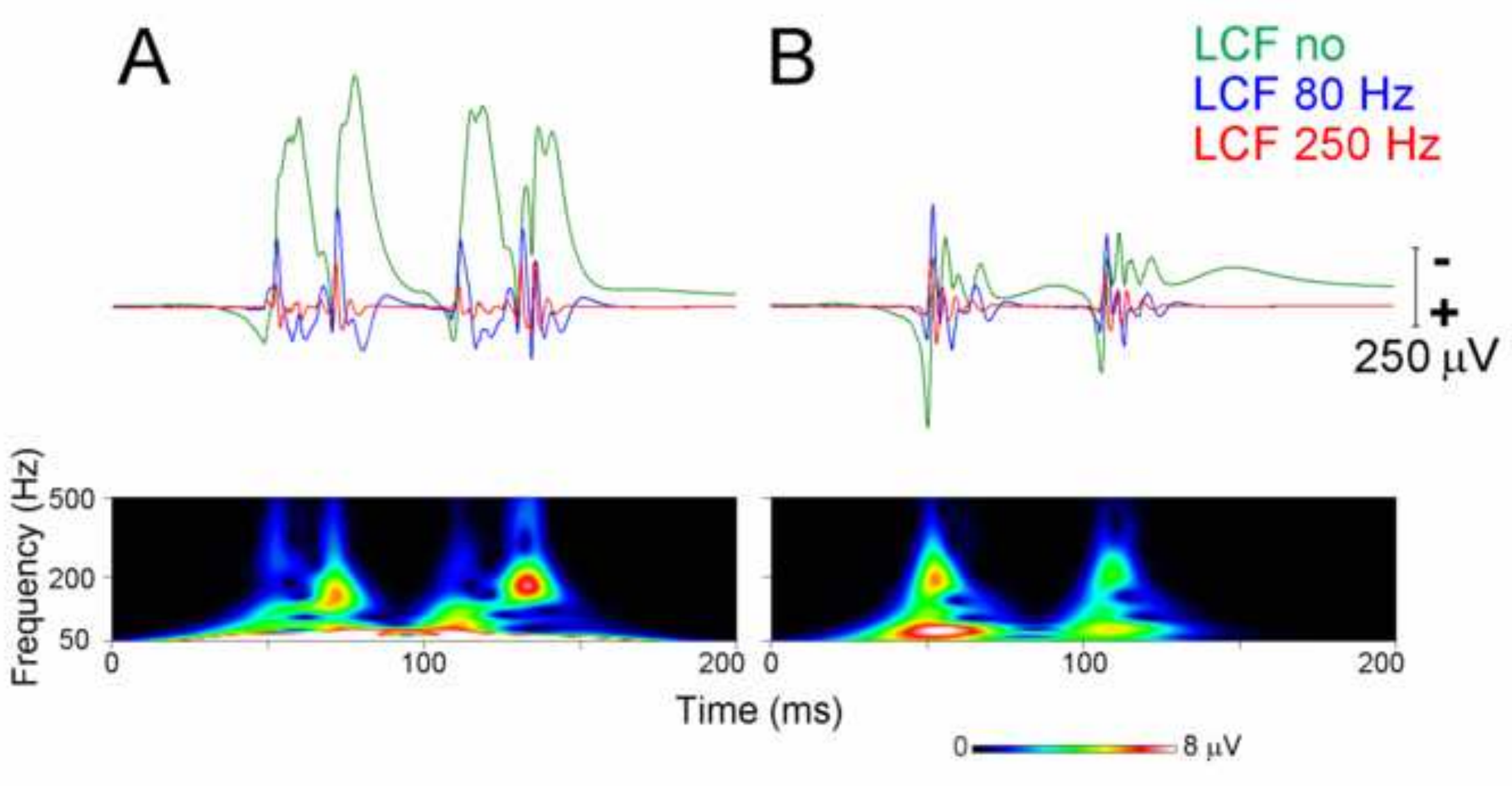

200

Time (ms)

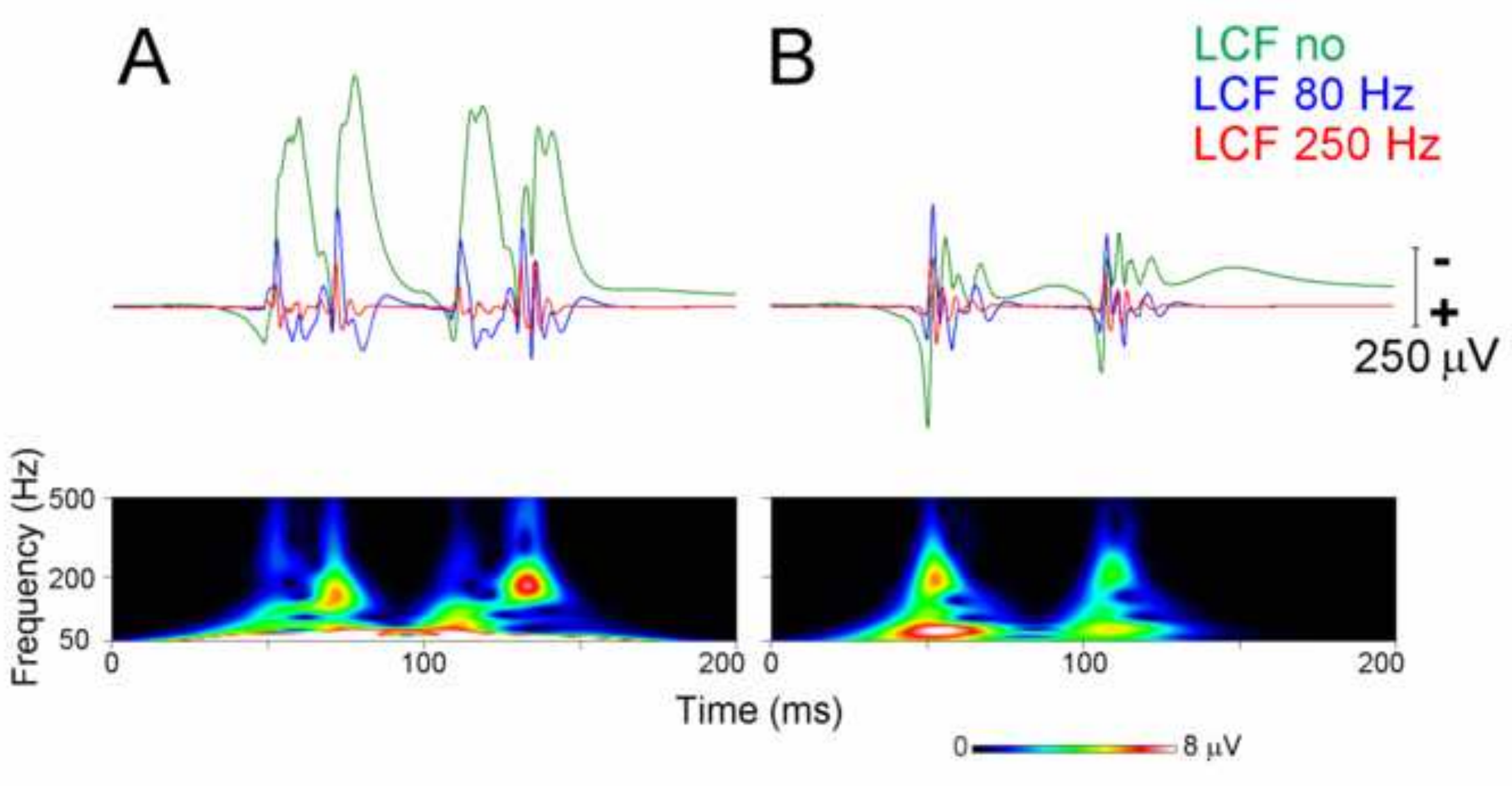

$8 \mu \mathrm{V}$
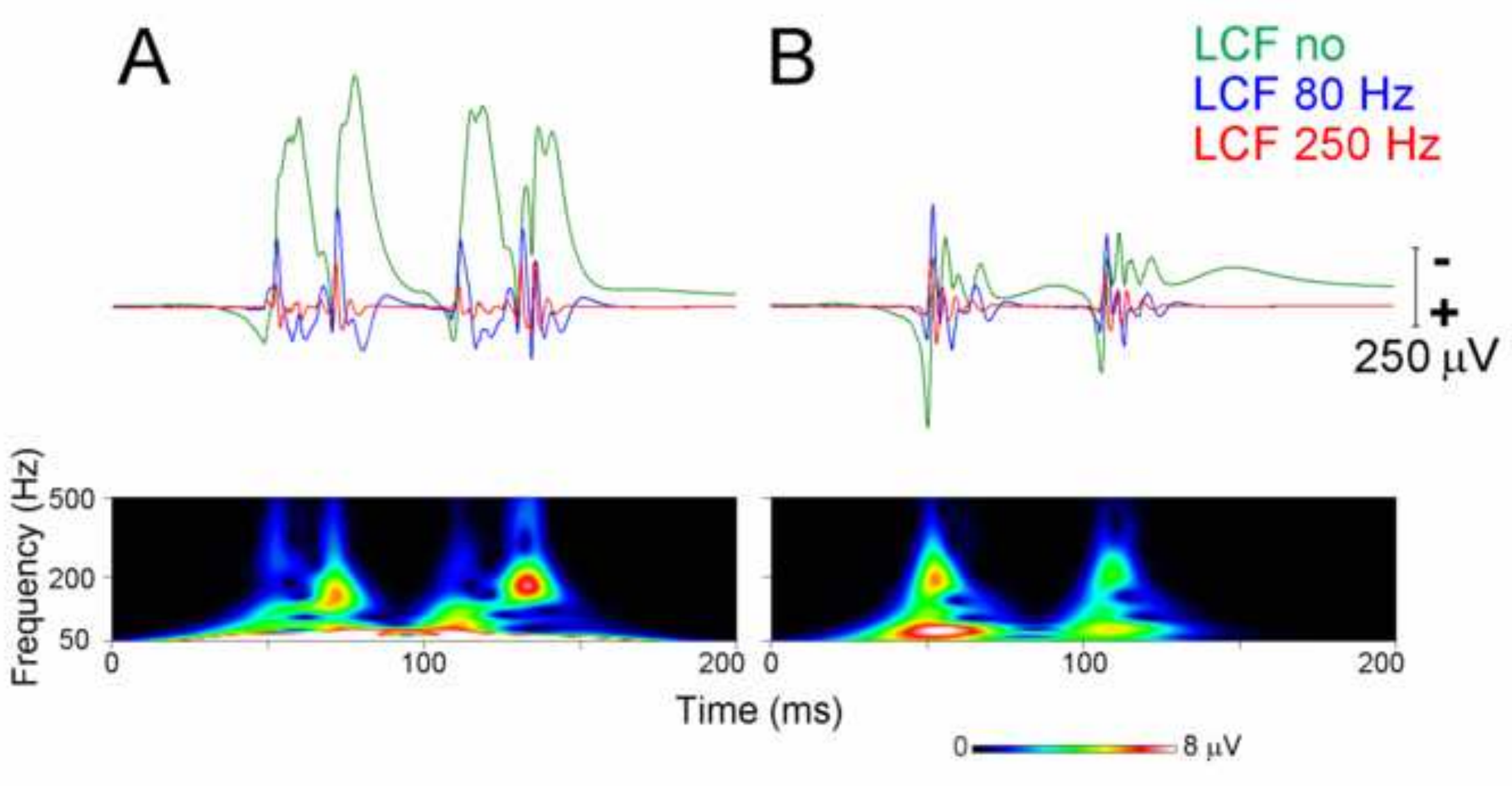